\title{
Developing Sensorimotor Countermeasures to Mitigate Postflight Locomotor Dysfunction
}

\author{
J.J. Bloomberg ${ }^{1}$, A.P. Mulavara ${ }^{2}$, H. Cohen ${ }^{3}$, C. A. Miller ${ }^{2}$, J.T. Richards ${ }^{2}$, J. Houser ${ }^{2}$, \\ P.V. McDonald ${ }^{2}$, R.D. Seidler ${ }^{4}$, L.A. Merkle ${ }^{5}$, G.E. Stelmach ${ }^{6}$ \\ ${ }^{1}$ NASA Johnson Space Center, Houston, TX,${ }^{2}$ Wyle Life Sciences Inc., Houston, TX, \\ ${ }^{3}$ Bobby R. Alford Department of Otorhinolaryngology and Communicative Sciences, \\ Baylor College of Medicine, Houston, TX, ${ }^{4}$ Brain Sciences Center, VA Medical Center, \\ Minneapolis, MN, ${ }^{5}$ Wingate University, Wingate, NC, ${ }^{6}$ Motor Control Laboratory, \\ Arizona State University, Tempe AZ
}

Following spaceflight, crewmembers experience postural and locomotor instability. The magnitude and duration of postflight sensorimotor disturbances increase with longer duration exposure to microgravity. These postflight postural and locomotor alterations can pose a risk to crew safety and to mission objectives if nominal or emergency vehicle egress is required immediately following long-duration spaceflight. Gait instabilities could prevent or extend the time required to make an emergency egress from the Orbiter, Crew Return Vehicle or a future Martian lander leading to compromised mission objectives. We propose a countermeasure that aids in maintaining functional locomotor performance. This includes retaining the ability to perform vehicular egress and meet early mission objectives soon after landing on a planetary surface.

At present, no operational countermeasure is available to mitigate the adaptive sensorimotor component underlying postural and locomotor disturbances that occur after spaceflight. Rather than develop a separate countermeasure, with commensurate demands on valuable crew time, we propose to develop an approach that can be easily integrated with the existing International Space Station (ISS) treadmill procedures. We suggest a unified, multi-disciplinary countermeasure system designed to enhance postflight adaptive locomotor function without requiring more commitment of limited crew resources. The goal of this study is to develop an inflight training regimen that enhances adaptive generalization of locomotor function, facilitating rapid and robust recovery of functional mobility after long-duration spaceflight.

The countermeasure we are developing is based on the concept of adaptive generalization training. In these training regimens practice is varied about some parameter, so that the subject learns to solve a class of motor problems, rather than a specific motor solution to one problem, i.e., the subject learns response generalizability or the ability to "learn to learn" under a variety of environmental constraints. Using this technique, we have proposed a countermeasure built around inflight treadmill exercise countermeasure activities. Subjects will locomote on the ISS treadmill while being exposed to variations in treadmill speed, body loading and visual input. Subjects will actively explore these transitions and learn to implement appropriate locomotor strategies during inflight treadmill exercise. By manipulating the sensorimotor conditions (speed, body load and visual input) during exercise we will systematically and repeatedly promote dynamic sensorimotor transitions in locomotor behavior, during inflight treadmill exercise. We 
anticipate that this training regimen will enhance locomotor response generalizability, facilitating locomotor adaptive transition from microgravity to partial (Mars) or unit (Earth) gravity environments following spaceflight. A series of supporting ground-based studies will be conducted to determined the optimal conditions required to produce dynamic transitions in locomotor patterns during treadmill exercise.

We will validate the efficacy of this countermeasure approach with long-duration ISS crewmembers. All subjects will be tested pre and postflight using testing techniques that we have used extensively in previous studies to characterize the effects of spaceflight on astronaut terrestrial locomotor control. Group 1, or controls, will have no exposure to the inflight adaptive generalization training protocol, to determine the effects of no intervention. These subjects will perform the nominal treadmill exercise procedures. They will be compared to Group 2, experimental subjects, who will be exposed to the adaptive generalization training protocol.

We envision that the next generation treadmill, used to support exploration class missions, will incorporate the use of a virtual reality (VR) system coupled with a multidirection treadmill that will allow the user to walk or run in any direction immersed in a varied and interesting virtual environment. This type of fusion interface, which integrally incorporates both virtual and non-virtual devices across sensory modalities, produces multi-sensory, virtually augmented, synthetic environments. These synthetic environments can serve as pre and inflight training tools providing sufficient sensorimotor and locomotor challenge to crewmembers to maximize their motor response adaptability, facilitating the adaptive transition to partial or unit gravity. In addition, a collateral benefit of the application VR technology, in this context, will be to make inflight training programs on long-duration spaceflight more interesting and challenging, ultimately leading to increased adherence to prescribed training regimens. 


\title{
Developing Sensorimotor Countermeasures to Mitigate Postflight Locomotor Dysfunction
}

\author{
J.J. Bloomberg ${ }^{1}$, A.P. Mulavara ${ }^{2}$, H. Cohen ${ }^{3}$, C. A. Miller ${ }^{2}$, J.T. Richards ${ }^{2}$, J. Houser ${ }^{2}$, \\ P.V. McDonald ${ }^{2}$, R.D. Seidler ${ }^{4}$, L.A. Merkle ${ }^{5}$, G.E. Stelmach ${ }^{6}$ \\ ${ }^{1}$ NASA Johnson Space Center, Houston, TX,${ }^{2}$ Wyle Life Sciences Inc., Houston, TX, \\ ${ }^{3}$ Bobby R. Alford Department of Otorhinolaryngology and Communicative Sciences, \\ Baylor College of Medicine, Houston, TX, ${ }^{4}$ Brain Sciences Center, VA Medical Center, \\ Minneapolis, MN, ${ }^{5}$ Wingate University, Wingate, NC, ${ }^{6}$ Motor Control Laboratory, \\ Arizona State University, Tempe AZ
}

Following spaceflight, crewmembers experience postural and locomotor instability. The magnitude and duration of postflight sensorimotor disturbances increase with longer duration exposure to microgravity. These postflight postural and locomotor alterations can pose a risk to crew safety and to mission objectives if nominal or emergency vehicle egress is required immediately following long-duration spaceflight. Gait instabilities could prevent or extend the time required to make an emergency egress from the Orbiter, Crew Return Vehicle or a future Martian lander leading to compromised mission objectives. We propose a countermeasure that aids in maintaining functional locomotor performance. This includes retaining the ability to perform vehicular egress and meet early mission objectives soon after landing on a planetary surface.

At present, no operational countermeasure is available to mitigate the adaptive sensorimotor component underlying postural and locomotor disturbances that occur after spaceflight. Rather than develop a separate countermeasure, with commensurate demands on valuable crew time, we propose to develop an approach that can be easily integrated with the existing International Space Station (ISS) treadmill procedures. We suggest a unified, multi-disciplinary countermeasure system designed to enhance postflight adaptive locomotor function without requiring more commitment of limited crew resources. The goal of this study is to develop an inflight training regimen that enhances adaptive generalization of locomotor function, facilitating rapid and robust recovery of functional mobility after long-duration spaceflight.

The countermeasure we are developing is based on the concept of adaptive generalization training. In these training regimens practice is varied about some parameter, so that the subject learns to solve a class of motor problems, rather than a specific motor solution to one problem, i.e., the subject learns response generalizability or the ability to "learn to learn" under a variety of environmental constraints. Using this technique, we have proposed a countermeasure built around inflight treadmill exercise countermeasure activities. Subjects will locomote on the ISS treadmill while being exposed to variations in treadmill speed, body loading and visual input. Subjects will actively explore these transitions and learn to implement appropriate locomotor strategies during inflight treadmill exercise. By manipulating the sensorimotor conditions (speed, body load and visual input) during exercise we will systematically and repeatedly promote dynamic sensorimotor transitions in locomotor behavior, during inflight treadmill exercise. We 
anticipate that this training regimen will enhance locomotor response generalizability, facilitating locomotor adaptive transition from microgravity to partial (Mars) or unit (Earth) gravity environments following spaceflight. A series of supporting ground-based studies will be conducted to determined the optimal conditions required to produce dynamic transitions in locomotor patterns during treadmill exercise.

We will validate the efficacy of this countermeasure approach with long-duration ISS crewmembers. All subjects will be tested pre and postflight using testing techniques that we have used extensively in previous studies to characterize the effects of spaceflight on astronaut terrestrial locomotor control. Group 1, or controls, will have no exposure to the inflight adaptive generalization training protocol, to determine the effects of no intervention. These subjects will perform the nominal treadmill exercise procedures. They will be compared to Group 2, experimental subjects, who will be exposed to the adaptive generalization training protocol.

We envision that the next generation treadmill, used to support exploration class missions, will incorporate the use of a virtual reality (VR) system coupled with a multidirection treadmill that will allow the user to walk or run in any direction immersed in a varied and interesting virtual environment. This type of fusion interface, which integrally incorporates both virtual and non-virtual devices across sensory modalities, produces multi-sensory, virtually augmented, synthetic environments. These synthetic environments can serve as pre and inflight training tools providing sufficient sensorimotor and locomotor challenge to crewmembers to maximize their motor response adaptability, facilitating the adaptive transition to partial or unit gravity. In addition, a collateral benefit of the application VR technology, in this context, will be to make inflight training programs on long-duration spaceflight more interesting and challenging, ultimately leading to increased adherence to prescribed training regimens. 